\title{
Geringfügig mehr Hirnblutungen unter SSRI
}

\begin{abstract}
Aufgrund der plättchenaggregationshemmenden Eigenschaften von SSRI (Selektive Serotonin-Wiederaufnahme-Hemmer) besteht ein erhöhtes Risiko für gastrointestinale Blutungen als unerwünschte Arzneimittelwirkung (UAW), insbesondere bei Komedikation mit gerinnungshemmenden Medikamenten. Eine Risikoerhöhung wird auch für intrakranielle Blutungen angenommen, doch bisherige Studien waren zum Teil widersprüchlich.
\end{abstract}

— Für die Metaanalyse wurden 16 prospektive Studien ausgewertet. Intrakranielle Blutungen waren mit SSRI-Behandlung assoziiert, und zwar sowohl in der nicht adjustierten (Rate Ratio [RR] $1,48)$ als auch in der adjustierten Analyse (RR 1,51), ebenso intrazerebrale Blutungen (nicht adjustiert: RR 1,68; adjustiert: RR 1,42).

In fünf der untersuchten Studien (drei zu intrakraniellen Blutungen und je eine zu hämorrhagischem Schlaganfall und intrazerebraler Blutung) wurde eine Risikoerhöhung bei einer Kombinationstherapie mit SSRI plus oralen Antikoagulanzien im Vergleich zu einer Therapie ausschließlich mit oralen Antikoagulanzien gefunden (RR 1,56).

Das Auftreten intrakranieller (ganz überwiegend intrazerebraler) Blutungen ist mit SSRI-Behandlung signifikant assoziiert, wobei allerdings aufgrund der Seltenheit dieser UAW in der Praxis ein eher geringes absolutes Risiko besteht.

\footnotetext{
- Hackam DG, Mrkobrada M.

Selective serotonin reuptake inhibitors and brain hemorrhage: a meta-analysis. Neurology 2012; 79: 1862-1865
}

\section{Kommentar}

Die jährliche Inzidenz von Hirnblutungen wird weltweit mit 24,6 pro 100000 Personen angegeben. Auf der Basis dieser Zahl und ihren Ergebnissen errechnen die Autoren der vorliegenden Studie, dass unter SSRI-Thera- pie mit je einer zusätzlichen intrazerebralen Blutung pro 10000 Personen gerechnet werden muss. Somit ergibt sich global ein sehr geringes absolutes Risiko trotz der klaren Assoziation.

Die Autoren der vorliegenden Studie fordern selbst weitere Studien mit größeren Stichprobenumfängen, um einzelne Subgruppen intrakranieller Blutungen besser abzugrenzen. Aufgrund dieser Studie sollte sicherlich keine allgemeine Änderung der generellen Verschreibungsgewohnheiten hinsichtlich SSRI erfolgen, jedoch sollte bei Patienten mit Risikofaktoren für intrakranielle Blutungen (vor allem orale Langzeitantikoagulation, frühere intrakranielle Blutung, zerebrale Amyloidangiopathie, schwere chronische Alkoholabhängigkeit, schwer kontrollierbare Hypertonie) eine sorgfältige Nutzen-RisikoAbwägung erfolgen und gegebenenfalls Antidepressiva aus anderen Klassen zur Behandlung erwogen werden.

C. LANGE-AsSCHENFELDT =

\section{Metformin hilft beim Ovarialkarzinom}

\section{Es gibt Hinweise, dass Metformin eine antikarzinogene Wirkung be- sitzt. Die Autoren fanden eine der- artige Wirkung von Metformin auch in experimentellen Studien beim Ovarialkarzinom.}

- An einer Fall-Kontroll-Studie nahmen Frauen mit Ovarialkarzinom teil, denen mindestens ein Jahr Metformin in einer Dosierung zwischen 500 und $2000 \mathrm{mg}$ täglich verordnet worden war. Als Kontrollen dienten Patientinnen mit Ovarialkarzinom, die kein Metformin erhalten hatten (nicht diabetische Kontrollen) und jene Frauen, die mit anderen Antidiabetika oder Insulin, nicht aber mit Metformin behandelt wurden (diabetische Kontrollen).

Diabetiker ohne Metforminbehandlung hatten - bei allerdings nur kleinen Fallzahlen - die niedrigste Lebenser- wartung (40\%) im Vergleich zu nichtdiabetischen Kontrollen (47\%) und den Patientinnen mit Metformin (67\%).

- Kumar S, Meuter A, Thapa P. et al. Metformin intake is associated with better survival in ovarian cancer. Cancer 2013; 119 : 555-562

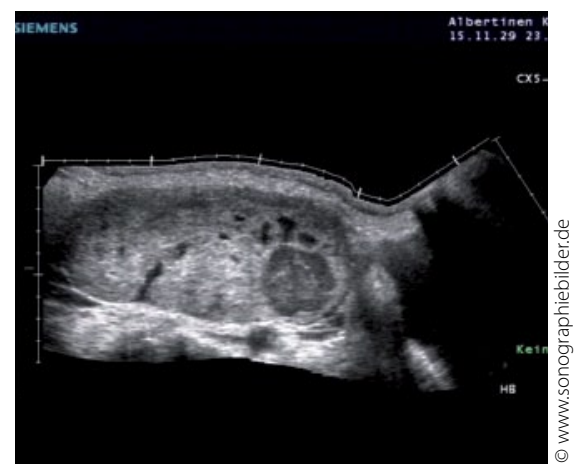

Großes, überwiegend solides Ovarialkarzinom.

\section{Kommentar}

Anlass der Studie waren experimentelle, epidemiologische und klinische Hinweise, nach denen Metformin bei verschiedenen Malignomen eine günstige Wirkung hat. Insofern kommt das Ergebnis der Studie nicht überraschend. Wahrscheinlich wird Metformin aber kein Bestandteil der Chemotherapie maligner Tumoren. Die Hoffnungen stützen sich darauf, dass jetzt die antitumoröse Zweitwirkung der Substanz durch Molekülveränderungen herausgefiltert, verstärkt und nutzbar gemacht werden könnte. Der Weg zur Zweitindikationen eines Arzneimittels ist schneller und kostengünstiger als die Entwicklung einer völlig neuen Substanz. Gerade Diabetologen wissen davon ein Lied zu singen: Die Sulfonamide, die zunächst als Chemotherapeutika gegen Krebs dienten, machten später als Antidiabetika und Diuretika Karriere.

H. HOLZGREVE = 